\title{
Lusioersily
}

\section{Beta-amyloid induced changes in A-type K+ current can alter hippocampo-septal network dynamics}

Zou, X., Coyle, D., Wong-Lin, K., \& Maguire, L. (2012). Beta-amyloid induced changes in A-type K+ current can alter hippocampo-septal network dynamics. Journal of Computational Neuroscience, 32(3), 465-477. https://doi.org/10.1007/s10827-011-0363-7

Link to publication record in Ulster University Research Portal

Published in:

Journal of Computational Neuroscience

Publication Status:

Published (in print/issue): 01/06/2012

DOI:

10.1007/s10827-011-0363-7

\section{Document Version}

Publisher's PDF, also known as Version of record

\section{General rights}

Copyright for the publications made accessible via Ulster University's Research Portal is retained by the author(s) and / or other copyright owners and it is a condition of accessing these publications that users recognise and abide by the legal requirements associated with these rights.

\section{Take down policy}

The Research Portal is Ulster University's institutional repository that provides access to Ulster's research outputs. Every effort has been made to ensure that content in the Research Portal does not infringe any person's rights, or applicable UK laws. If you discover content in the Research Portal that you believe breaches copyright or violates any law, please contact pure-support@ulster.ac.uk. 


\title{
Beta-amyloid induced changes in A-type $\mathrm{K}^{+}$current can alter hippocampo-septal network dynamics
}

\author{
Xin Zou • Damien Coyle • KongFatt Wong-Lin • \\ Liam Maguire
}

Received: 19 June 2011 / Revised: 10 August 2011 /Accepted: 8 September 2011

(C) Springer Science+Business Media, LLC 2011

\begin{abstract}
Alzheimer's disease (AD) progression is usually associated with memory deficits and cognitive decline. A hallmark of $\mathrm{AD}$ is the accumulation of beta-amyloid $(\mathrm{A} \beta)$ peptide, which is known to affect the hippocampal pyramidal neurons in the early stage of AD. Previous studies have shown that $\mathrm{A} \beta$ can block A-type $\mathrm{K}^{+}$currents in the hippocampal pyramidal neurons and enhance the neuronal excitability. However, the mechanisms underlying such changes and the effects of the hyper-excited pyramidal neurons on the hippocampo-septal network dynamics are still to be investigated. In this paper, A $\beta$-blocked A-type current is simulated, and the resulting neuronal and network dynamical changes are evaluated in terms of the theta band power. The simulation results demonstrate an initial slight but significant theta band power increase as the A-type current starts to decrease. However, the theta band power eventually decreases as the A-type current is further decreased. Our analysis demonstrates that $\mathrm{A} \beta$ blocked A-type currents can increase the pyramidal neuronal excitability by preventing the emergence of a steady state. The increased theta band power is due to more pyramidal neurons recruited into spiking mode during the peak of pyramidal theta oscillations. However, the decreased theta band power is caused by the spiking phase relationship between different neuronal populations, which is critical for theta oscillation, is violated by the hyper-excited pyramidal neurons. Our findings could provide potential implications on some $\mathrm{AD}$ symptoms, such as memory deficits and AD caused epilepsy.
\end{abstract}

\section{Action Editor: N. Kopell}

X. Zou $(\bowtie) \cdot$ D. Coyle $\cdot$ K. Wong-Lin $\cdot$ L. Maguire Intelligent Systems Research Centre, University of Ulster, Magee Campus,

Derry, Northern Ireland, UK, BT48 7JL

e-mail: x.zou@hotmail.co.uk
Keyword Alzheimer's disease $\cdot \beta$-Amyloid - A-type $\mathrm{K}^{+}$ current $\cdot$ Phase-plane analysis $\cdot$ Hippocampo-septal theta rhythm

\section{Introduction}

Alzheimer's disease (AD) is a neurodegenerative disease associated with memory deficits and cognitive decline, which are caused by pathological changes in the brain (Minati et al. 2009). In the early stage of $\mathrm{AD}$, the hippocampus in the medial temporal lobe is one of the first regions to be affected, especially the hippocampal pyramidal neurons (Adeli et al. 2005; Li et al. 2010). Damage to the hippocampus can lead to profound amnesia. Hippocampo-septal theta rhythm $(4-7 \mathrm{~Hz})$ has been found to play a critical role in memory processing, and theta rhythm abnormalities is usually related to memory deficits and pathological changes in brain (Vertes 2005; Colom 2006). Therefore, we will evaluate the hippocampus dynamical abnormalities in terms of the hippocamposeptal theta band power changes.

$\mathrm{AD}$ is characterized by two neuropathological structures: neurofibrillary tangles and senile plaques (Tiraboschi et al. 2004). Neurofibrillary tangles are caused by the microtubule-binding protein, tau, becoming hyperphosphorylated. Senile plaques are mainly composed of betaamyloid $(A \beta)$. $A \beta$ acts as a neurotoxin causing neuronal dysfunction and apoptosis (Hardy and Higgins 1992) and synaptic plasticity impairment (Holscher et al. 2007). A $\beta$ may change the activity of various ionic channels in pyramidal neurons, e.g., L-type $\mathrm{Ca}^{2+}$ channels $\left(\mathrm{I}_{\mathrm{Ca}}\right)$ (Webster et al. 2006); A-type fast-inactivating $\mathrm{K}^{+}$channels $\left(\mathrm{I}_{\mathrm{A}}\right)$ and delayed rectifying $\mathrm{K}^{+}$channels $\left(\mathrm{I}_{\mathrm{K}}\right)$ (Good et al. 1996); large-conductance $\mathrm{Ca}^{2+}$-activated $\mathrm{K}^{+}$channels $\left(\mathrm{I}_{\mathrm{CT}}\right)$ 
(Ye et al. 2010; Chi and Qi 2006). In addition, $A \beta$ can disturb the neuromodulators, (Tran et al. 2002; Palop and Mucke 2010), which control memory encoding and recall (Hasselmo et al. 1996). As A $\beta$ precedes tau protein in the progression of $\mathrm{AD}$ (Takahashi et al. 2010), this work focuses on the study of the impact of $A \beta$ on the hippocampus.

The aim of this paper is to investigate the impacts of $A \beta$ induced pathological changes on hippocampal neural dynamics through computational studies. Extensive computational work has been performed to investigate the AD-induced hippocampal memory encoding, retrieval difficulties and pathological changes in synapses (Adeli et al. 2005). Building on previous work, we have constructed an integrated computational model of the hippocampal CA1 region coupled to the medial septum, which includes pyramidal, basket, OLM and MSGABA neurons. We identify the significance of the impact of $A \beta$ on the hippocampal network dynamics by evaluating changes in the hippocampo-septal theta band power. In our previous work, we have evaluated various ionic channels in the hippocampal pyramidal neurons, which have been reported to be affected by $\mathrm{A} \beta$. Specifically, changes in $\mathrm{I}_{\mathrm{Ca}}, \mathrm{I}_{\mathrm{A}}, \mathrm{I}_{\mathrm{K}}$ and $\mathrm{I}_{\mathrm{CT}}$ have been evaluated and we found that only $A \beta$-blocked $I_{A}$ has induced a significant theta band power change (Zou et al. 2011), probably due to the increased pyramidal neuronal excitability (Morse et al. 2010). In this paper, a wider range of model parameters of $\mathrm{I}_{\mathrm{A}}$ will be explored and the corresponding theta band power changes will be evaluated. Then the mechanisms underlying the $\mathrm{A} \beta$-blocked $\mathrm{I}_{\mathrm{A}}$ induced pyramidal neuronal excitability increases and its relationship to the theta band power changes will be mathematically and systematically investigated. We will also explore possible implications of our results to memory disorder and epilepsy.

\section{Method}

\subsection{Conductance-based neural network}

We construct a hippocampo-septal computational model based on the Hodgkin-Huxley type formalisms with an architecture following the work in (Hajós et al. 2004). The model incorporates three types of neurons from the hippocampus CA1, i.e., pyramidal, basket and OLM neurons and one type of neuron from the medial septum, i.e., MSGABA. These neurons have been demonstrated to contribute to the theta rhythm activity in in vivo experiments (Csicsvari et al. 1999; Ylinen et al. 1995; Klausberger et al. 2003) and simulations (Wang 2002; Rotstein et al. 2005). The schematic illustration of the network architecture is shown in Fig. 1. Each type of neuron in Fig. 1 represents a population of identical neurons. The network theta oscillations are generated from the intrinsic theta oscillations of

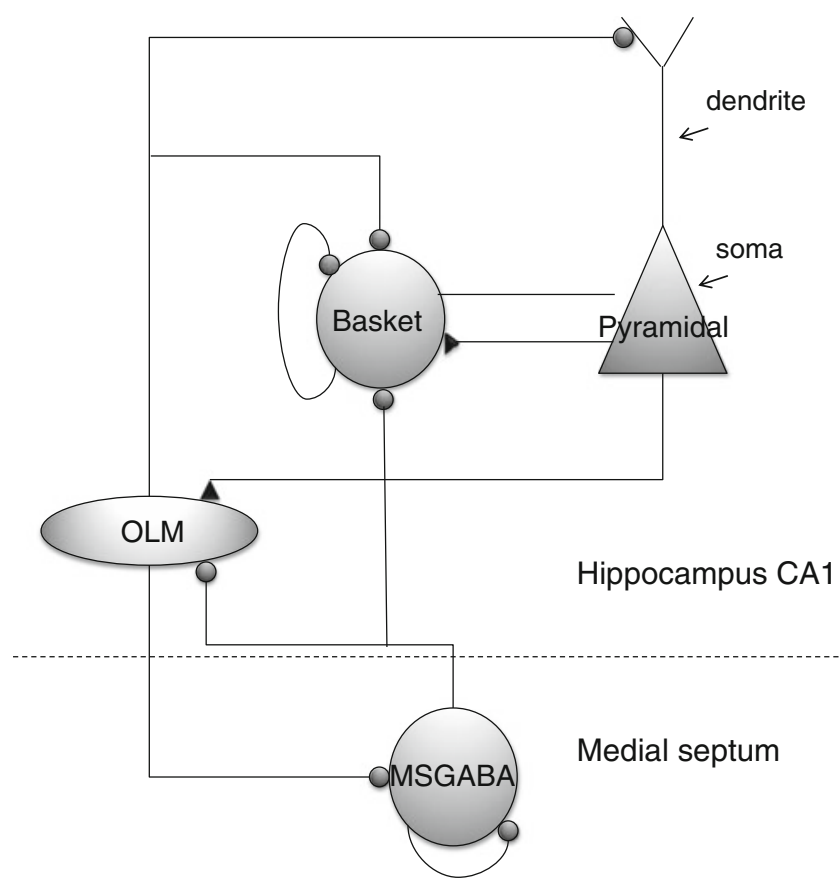

Fig. 1 Hippocampo-septal network model. The network consists of four types of neuronal populations, i.e., pyramidal, basket, OLM and MSGABA neurons. The inhibitory $\mathrm{GABA}_{\mathrm{A}}$ synaptic connections are indicated by ' $\bullet$ ', and the excitatory AMPA and NMDA synaptic connections are indicated by ' $\boldsymbol{\Delta}$ '

MSGABA neurons (Stewart and Fox 1990; Cobb et al. 1995; Wang 2002; Freund and Antal 1988; Toth et al. 1997).

The pyramidal neuron model consists of two compartments, one for the soma and the other for the dendrite. Subject to the model proposed in (Wang 1998), the soma compartment has spike generating currents $\mathrm{I}_{\mathrm{Na}}$ and $\mathrm{I}_{\mathrm{K}}$ and the dendrite contains a calcium dependent potassium current $\mathrm{I}_{\mathrm{AHP}}$. Both the soma and the dendrite contain leakage currents $I_{L}$ and high-threshold L-type calcium currents $\mathrm{I}_{\mathrm{Ca}}$. Furthermore, the pyramidal neurons in the hippocampus CA1 contain other types of ionic currents to account for neuronal functions (Warman et al. 1994). In this work, we will incorporate some of those currents which have been shown to be affected by $A \beta$. As a result, our model also contains A-type transient potassium currents $\mathrm{I}_{\mathrm{A}}$ and large-conductance calcium dependent potassium currents $\mathrm{I}_{\mathrm{CT}}$ in the soma and dendrite, respectively. The membrane potential dynamics are modelled as follows:

$\dot{V}_{s}=-I_{L}-I_{N a}-I_{K}-I_{C a}-I_{A}-I_{C T}-\frac{g_{c}}{p}\left(V_{s}-V_{d}\right)-I_{s y n, s}+I$

$\dot{V}_{d}=-I_{L}-I_{C a}-I_{A H P}-I_{A}-I_{C T}-\frac{g_{c}}{1-p}\left(V_{d}-V_{s}\right)-I_{s y n, d}$ 
where $V$ denotes membrane potential, subscript $s$ and $d$ denote soma and dendrite, respectively. The synaptic current $I_{s y n}$ represents the synaptic interactions from other neurons in the network. $g_{c}$ is the coupling conductance between soma and dendrite, and $p=$ soma area/total area, with 0.5 as the default value. To emulate heterogeneity in the brain, the injected DC current, $I$, for each neuron is not chosen to be identical. This is achieved by allowing $I$ to follow a Gaussian distribution with mean $I_{\mu}$ and standard derivation $I_{\sigma}$. For the pyramidal neuronal population, $I_{\mu}=$ $4.9 \mu \mathrm{A} / \mathrm{cm}^{2}$ and $I_{\sigma}=0.1 \mu \mathrm{A} / \mathrm{cm}^{2}$.

The one-compartmental models of the other three neuronal types are constructed in the same way as in (Wang 2002; Wang and Buzsaki 1996). The model of an OLM neuron has $I_{N a}, I_{K}, I_{L}, I_{C a}$, hyperpolarization activated current $I_{h}$ and $I_{A H P}$ Eq. (3). The model of a basket neuron has $I_{N a}, I_{K}$, and $I_{L}$ Eq. (4). The MSGABA neuronal model contains $I_{N a}, I_{K}, I_{L}$ and a slowly inactivating potassium current $I_{K S}$ Eq. (5).

$$
\begin{aligned}
& \dot{V}_{O L M}=-I_{L}-I_{N a}-I_{K}-I_{C a}-I_{h}-I_{A H P}-I_{s y n}+I \\
& \dot{V}_{\text {Basket }}=-I_{L}-I_{N a}-I_{K}-I_{s y n}+I \\
& \dot{V}_{M S G A B A}=-I_{L}-I_{N a}-I_{K}-I_{K S}-I_{s y n}+I
\end{aligned}
$$

For the basket, OLM and MSGABA neuronal populations, $I_{\mu}=1.4 \mu \mathrm{A} / \mathrm{cm}^{2}, 0 \mu \mathrm{A} / \mathrm{cm}^{2}, 2.2 \mu \mathrm{A} / \mathrm{cm}^{2}$, respectively; $I_{\sigma}=$ $0.1 \mu \mathrm{A} / \mathrm{cm}^{2}$ for all populations. Details of the definition of all the other parameters are provided in the Appendix.

We found from our simulations that the changes in the connection topology do not significantly affect the dynamics of the network and our results, as far as the number of synapses per cell is larger than a critical value (Wang and Buzsaki 1996). Therefore, the simulation results presented in this paper are based on an all-to-all connectivity, because the exclusion of heterogeneity in the connectivity makes our analysis more tractable. The pyramidal neurons innervate basket neurons via AMPA-type receptor and OLM via AMPA- and NMDA-type receptors, the other synaptic connections are mediated by the $\mathrm{GABA}_{\mathrm{A}}$-type receptors. The number of pyramidal, basket, OLM and MSGABA neurons is $10,100,30$ and 50 , respectively. The ratio of interneurons is based on that reported in (Freund and Buzsaki 1996). Although the number of pyramidal neurons is large in the hippocampus, a limited number of pyramidal neurons were used in (Hajós et al. 2004) for the purpose of computational efficiency. It has been shown that such limited numbers of pyramidal neurons are sufficient to yield acceptable statistics and thus this setting is used in our work. Noise in the membrane potential is generated by following a Gaussian distribution with zero mean and standard derivation of $1.1 \mu \mathrm{A} / \mathrm{cm}^{2}$. The membrane noise is randomly generated in each trial. Note that membrane noise is excluded in Section 3.2 for analysis.

In this work, network oscillations in theta band are investigated by evaluating the summed membrane potentials of the neurons (Pedroarena and Llinas 1997) (this is equivalent to using a summation of synaptic currents as illustrated in Fig. 2). The relative power of theta band ( $\%$ of the total power) is obtained by a fast Fourier transform with a $2 \mathrm{~s}$ length Hanning window. The presented result for each group of parameters is obtained from simulations averaged over several trials, each trial lasts for $6 \mathrm{~s}$. In each group, we include 15 individual trials (we have found through extensive experimentation that using a higher number of trials does not alter the obtained average theta band power). The statistical difference between groups is evaluated using a one-way ANOVA test. The results obtained in various conditions are compared with the results obtained in the original condition, and any significant difference between the two groups is indicated by $p<0.05$.

\subsection{A reduced pyramidal neuron model for neuronal dynamical analysis}

A useful way to analyze a dynamical (neural) system is to construct its phase plane (also known as phase portrait) (Izhikevich 2007). A phase plane can depict the stability of the system such that its dynamics can be rigorously analyzed and understood geometrically. However, the limitation of the phase-plane analysis is that it is difficult to be applied for a system that contains multiple dynamical variables. The complexity of the two-compartmental model of pyramidal neurons in the network makes it unsuitable to be analyzed using phase-plane analysis, because it contains too many dynamical variables.

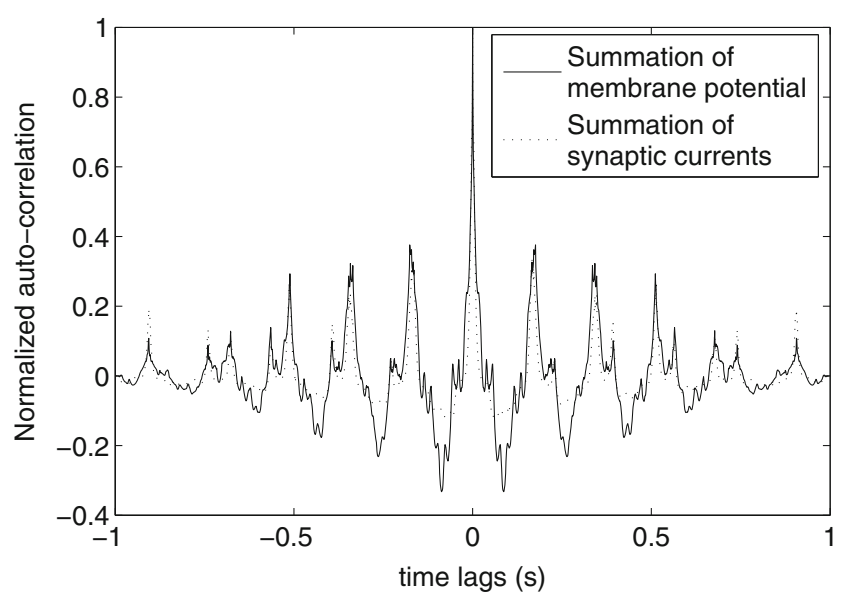

Fig. 2 Auto-correlations of summation of membrane potential (solid line) and summation of synaptic currents (dashed line). Measuring either produces the same pattern of oscillation 
To address this issue, the pyramidal neuronal model is reduced by maintaining as few as ionic currents and compartments, but at the same time keeping the network dynamics unaffected. As a result, our reduced pyramidal neuronal model is a one-compartmental model that consists of $I_{N a}, I_{K}$ and $I_{L}$ from the soma and $I_{A}$ from the dendrite in the full model. The dynamical variables in the reduced one-compartmental pyramidal model can be further reduced by performing additional approximations. First, as the activation gating process of $I_{N a}$ and $I_{A}$, denoted by $m$ and $a$, respectively, are very fast, they can be considered as being relatively instantaneous, therefore their steady-state value $m_{\infty}$ and $a_{\infty}$ can be used. Second, there is an approximately linear relationship between the inactivation gating variable of $I_{\mathrm{Na}}$, $h$, and the activation gating variable of $I_{K}, n$, i.e., $h=0.89$ 1.In (Izhikevich 2007). Based on the above approximations, the final reduced dynamics of the pyramidal neuron becomes:

$$
\begin{aligned}
\dot{V}_{p y r} & =I-I_{L}-I_{N a}-I_{K}-I_{s y n}-I_{A} \\
& =I-g_{L}\left(V-E_{L}\right)-g_{N a} m_{\infty}^{3}(0.89-1.1 n)\left(V-E_{N a}\right) \\
& -g_{K} n^{4}\left(V-E_{K}\right)-g_{A} a_{\infty} b\left(V-E_{K}\right)
\end{aligned}
$$

Note that Eq. (6) only consists of three dynamical variables, i.e., $V, n$ and $b$. The definition of the parameters is the same as they are defined in Eqs. (1 and 2) (see the Appendix for further details).

The aim of the model reduction approximation is to reduce the number of dynamical variables but not to significantly change the system statistical characteristics. To verify that the reduced model dynamics do not deviate significantly from the full model, results from 15 trials running with original parameter settings were obtained for both the full network and the network employing the reduced pyramidal neuron model implementations. To compensate for the changes in the pyramidal neurons model, $I_{\mu}$ are modified as $3.5 \mu \mathrm{A} / \mathrm{cm}^{2},-0.4 \mu \mathrm{A} / \mathrm{cm}^{2}, 1 \mu \mathrm{A} /$ $\mathrm{cm}^{2}$ and $2.2 \mu \mathrm{A} / \mathrm{cm}^{2}$ for pyramidal, OLM, basket and MSGABA populations, respectively. A one-way ANOVA test was performed and the results verify that the model reduction process does not significantly change theta band power $(p=0.6557)$. Note that the simplified pyramidal neuronal model is applied in the phase-plane analysis presented in Section 3.2.1.

\section{Results}

\subsection{Network simulations}

The constructed network model produces theta rhythm activity as observed in the hippocampus CA1 and medial septum. The theta rhythm activity is characterized by phasic recurrent spikes clustering in different neuronal popula- tions. A theta rhythm period can be split into three time epochs, as illustrated in Fig. 3.

- $T_{1}$ : Early in time epoch $\mathrm{T}_{1}$, the pyramidal neurons start to spike which evoke the OLM and basket neurons quickly via fast AMPA-type receptors. The subsequent spikes of the OLM neurons are promoted by the NMDA-type receptors, which in turn suppress the pyramidal and basket neurons. After the OLM neurons have spiked, the basket and pyramidal neurons are gradually depolarized by the injected depolarization currents.

- $T_{2}$ : As the basket neurons depolarize faster than the pyramidal neurons, they will spike earlier and inhibit the pyramidal neurons via the $\mathrm{GABA}_{\mathrm{A}}$-type receptors. During the course of time epoch $T_{2}$, the basket neurons keep spiking and the pyramidal neurons are hyperpolarized.

- $T_{3}$ : At the beginning of epoch $T_{3}$, the MSGABA neurons start to spike, which inhibit basket neurons and release the pyramidal neurons. The spikes of the MSGABA neurons are stopped by the firing of OLM neurons that have been evoked by the pyramidal neurons.

Such a spiking phase relationship is consistent with previous experimental and computational studies. In (Klausberger et al. 2003; Cutsuridis et al. 2010; Klausberger and Somogyi 2008), it has been shown that the pyramidal and OLM neurons spike in phase, while the basket neurons spike out of

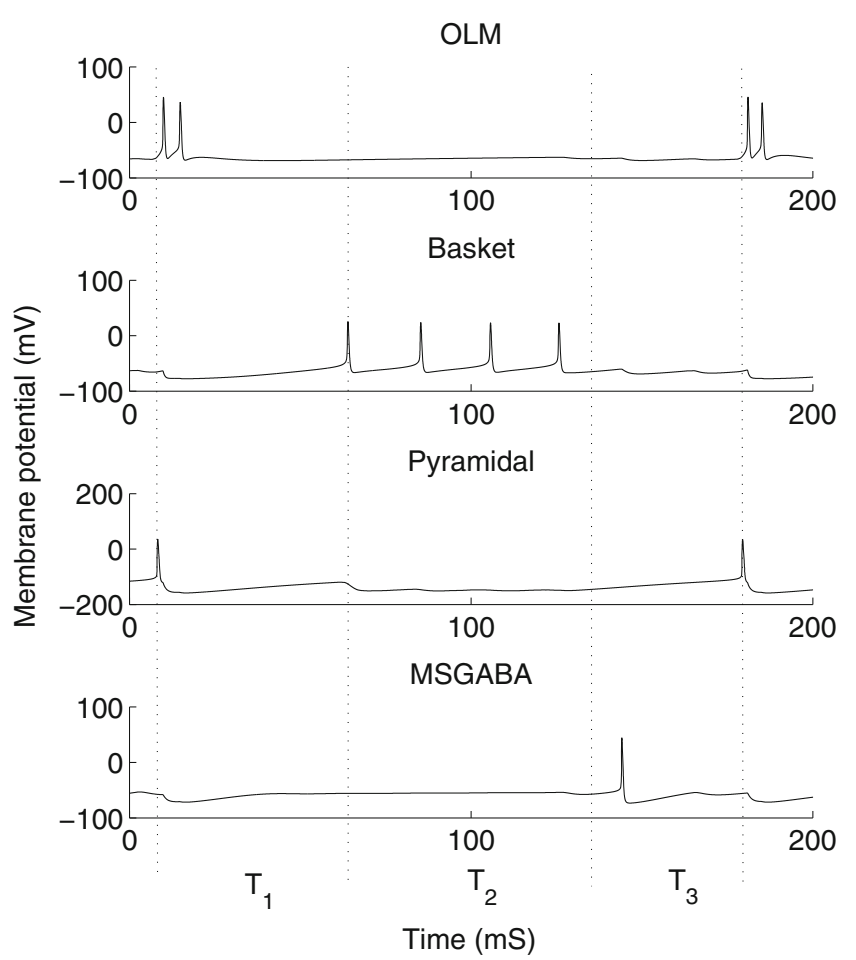

Fig. 3 Multiple phases of spiking dynamics constitute the network theta oscillation. Each theta oscillation consists of three time epochs, denoted by $T_{1}, T_{2}$ and $T_{3}$. The dynamics are obtained using Eqs. (15). This is obtained in the noise-free condition 
phase with them. Wang (2002) has shown that the OLM and MSGABA neurons spike out of phase in theta oscillation.

In this work, the maximum conductance $\mathrm{g}_{\mathrm{A}}$ in dendrite is decreased to simulate the $A \beta$-blocked $\mathrm{I}_{\mathrm{A}}$ in the dendrite (Chen 2005; Morse et al. 2010; Good et al. 1996; Xu et al. 1998; Zhang and Yang 2006). The obtained theta band power with different values of $g_{A}$ is illustrated in Fig. 4. Interestingly, it can be seen that with the decrease in $g_{A}$, the theta band power first slightly increases to a maximum value with $0.9 \mathrm{~g}_{\mathrm{A}}$ before it starts to decrease. When $\mathrm{g}_{\mathrm{A}}$ is below $0.3 \mathrm{~g}_{\mathrm{A}}$, the theta band power almost disappears. Figure 4 demonstrates that decreasing $\mathrm{g}_{\mathrm{A}}$ initially causes an increase in theta oscillations, after which a further decrease in $\mathrm{g}_{\mathrm{A}}$ produces a corresponding decrease in the theta activity. To understand the network's dynamics underlying such observations, the dynamics of a single reduced pyramidal neuron and the whole network are systematically analyzed in Section 3.2.

\subsection{Mechanisms underlying the changes}

in the network dynamics

The simulation results presented in the previous section show that decreased $g_{A}$ induces theta band power changes. In our previous work (Zou et al. 2011), we have shown that the increased theta band power is due to the increased synchrony of the pyramidal neuronal population. The network theta oscillation is originated from MSGABA neurons, and the theta oscillation of pyramidal neurons is driven by the inhibitory post synaptic currents from the interneurons. During the trough of a pyramidal theta cycle, the inhibition on the pyramidal neurons is large; therefore

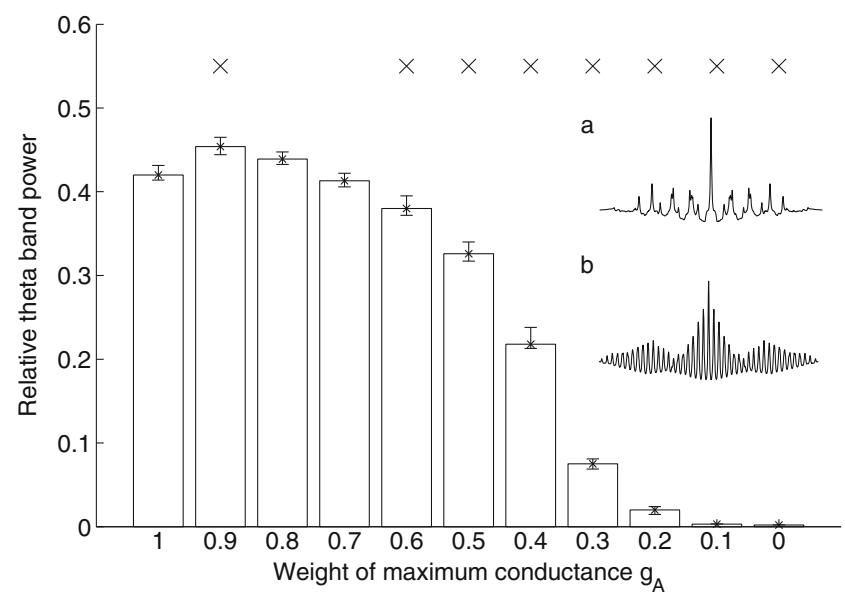

Fig. 4 Changes in theta band power with decreasing $\mathrm{g}_{\mathrm{A}}$. Each presented theta band power is averaged from 15 trials. ' $X$ ' indicates the average theta band power is significantly different from that obtained in the original $\mathrm{g}_{\mathrm{A}}$ condition $(p<0.05)$. Error bars are standard errors. Insets: autocorrelations of the summation of membrane potentials, a) original $\left.g_{A}, b\right) 0.2 g_{A}$. Time duration: $1 \mathrm{~s}$ the pyramidal neurons are mostly quiescent. But during the peak or crest of the pyramidal theta cycle, as inhibition from the interneurons decreases, the pyramidal neurons will be able to spike; and the neuronal dynamics (to spike or not) is dependent on the received depolarization currents and the neuronal excitability. As can be inferred from Fig. 3 (epoch T3) that the inhibition afference is low and relatively constant during this time epoch, the pyramidal neuronal dynamics are mainly dependent on the intrinsic neuronal excitability. The higher the neuronal excitability is, the easier the pyramidal neurons can spike (resulting in higher population synchrony). Therefore, we are justified to use a reduced single pyramidal neuron model to analyze the pyramidal neuronal excitability. This will be performed in Section 3.2.1. Furthermore, the relationship between the hyper-excitability of pyramidal neurons and the decreased theta band power will be investigated in Section 3.2.2.

\subsubsection{A -blocked $I_{A}$ increases neuronal excitability}

To investigate the enhanced neuronal excitability as a result of decreased $g_{A}$, a dynamical systems analysis of a single reduced pyramidal neuron modelled by Eq. (6) was performed. The applied DC current, $I$, in Eq. (6) is reduced to $2 \mu \mathrm{A} / \mathrm{cm}^{2}$ to compensate for the absence of inhibition from interneurons. As mentioned in Section 2.2, the reduced pyramidal neuron model contains three dynamical variables $V, n$ and $b$. Therefore, the time evolution of its state can be plotted as trajectories in a three-dimensional (phase) space-a stereoscope image (Izhikevich 2007).

To demonstrate the dynamical changes more clearly, we will take $0.8 \mathrm{~g}_{\mathrm{A}}$ as an example in this section instead of using $0.9 \mathrm{~g}_{\mathrm{A}}$. Figure 5 plots the (noiseless) trajectories in the original and $0.8 \mathrm{~g}_{\mathrm{A}}$ conditions, with either a high or low initial value of its membrane potential $V_{0}$. In Fig. 5, the initial values of $b$ and $n$ are all zero. Figure 5 ( $\mathrm{a}$ and $\mathrm{b}$ ) are obtained in the original $\mathrm{g}_{\mathrm{A}}$ condition; $\mathrm{c}$ and $\mathrm{d}$ are obtained with the value of $g_{A}$ set at 0.8 times its original value. From the plots, it can be clearly seen that in the original $\mathrm{g}_{\mathrm{A}}$ condition, the tendency to spike (an increase followed by a decrease in $V$ ) is highly dependent on its initial state. With a low initial membrane potential value of $V_{0}=-65 \mathrm{mV}$, the neuron will quickly converge to a stable state without spiking (Fig. 5(a)). If the initial membrane potential value is higher $\left(V_{0}=-45 \mathrm{mV}\right)$, the neuron produces a spike and then converges back to the stable state (Fig. 5(b)). In the case of $0.8 \mathrm{~g}_{\mathrm{A}}$, the dynamics of the neuron become less dependent on its initial state. In Fig. 5 (c and d), the neuron is capable of spiking with either a high or low initial membrane potential. Note that it also spikes repetitively.

Under the original $\mathrm{g}_{\mathrm{A}}$ condition and in the presence of noise, the probability of a pyramidal neuron spiking is reduced by the presence of the stable state. However in the 


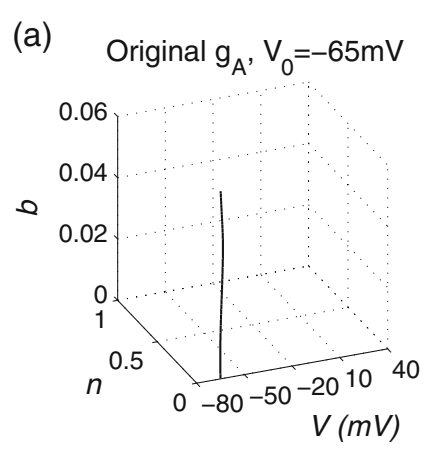

(b)

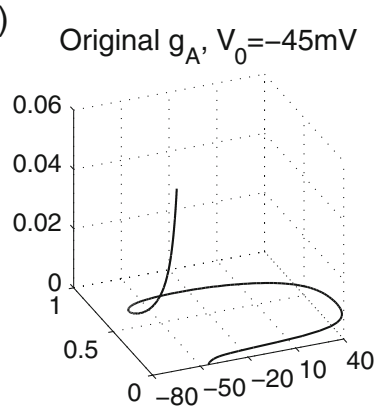

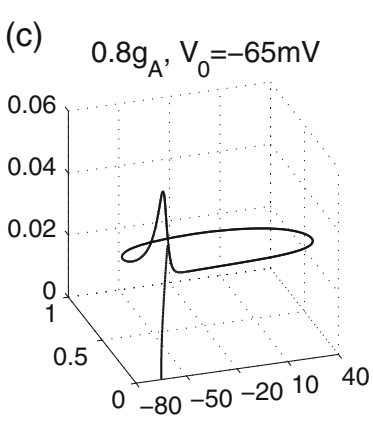

(d)

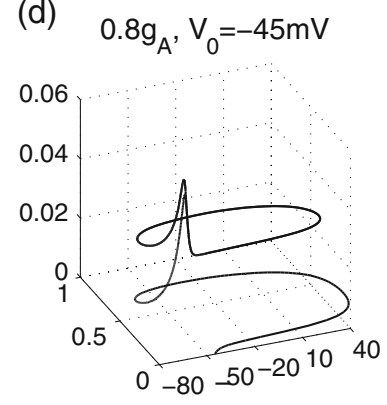

Fig. 5 Stereoscope images of the trajectories of pyramidal neuronal dynamics in the three-dimensional phase space $V, n$ and $b$. Plots (b-d) have the same axes labelling as Plot (a). Plots (a) and (b) are obtained in the original $\mathrm{g}_{\mathrm{A}}$ condition; (c) and (d) are obtained in the $0.8 \mathrm{~g}_{\mathrm{A}}$ condition. The trajectory in (a) indicates no spike (the membrane potential $V$ does not increase to positive then return to negative), (b) indicates single spike, (c) and (d) are repetitive spikes. The trajectories in (a) and (b) terminate at a stable state; however, trajectories in (c) and (d) do not have termination. $V_{0}$ is initial value of $V$. The initial values of $n$ and $b$ are all zero

case of low $g_{A}$, pyramidal neurons become more susceptible to spiking as there is no such stable state in the system. This explains the increased excitability of pyramidal neurons with low $g_{A}$. Although Fig. 5 shows some principle dynamical characteristics of the pyramidal neurons associated with a decrease in $g_{A}$, it is more rigorous to visualize and analyze the above mechanisms in a two-dimensional phase space. For example, the transition from resting (Fig. 5(a)) to repetitive spiking (Fig. 5(c)) could be induced by a bifurcation (abrupt qualitative change in behaviour). To clearly demonstrate the properties of such a bifurcation, a phase-plane analysis is employed by producing twodimensional slices of the stereoscope images from Fig. 5.

We observe that the dynamics of $b$ is an order of magnitude slower than $n$ at low membrane potential and almost unchanged at high membrane potential. Therefore a two-dimensional slice of the stereoscope image can be approximated by fixing $b$ at a specific value, and plotting the dynamical trajectories in the $(V, n)$ space. Nullclines in the $(V, n)$ space, i.e., $V$ - and $n$-nullclines, are obtained by separately solving two equations, $V=0$ and $\dot{n}=0$, such that

$$
\begin{aligned}
\dot{V}= & 2-g_{L}\left(V-E_{L}\right)-g_{N a} m_{\infty}^{3}(0.89-1.1 n)\left(V-E_{N a}\right) \\
& -g_{K} n^{4}\left(V-E_{K}\right)-g_{A} a_{\infty} b\left(V-E_{K}\right)=0 \\
\dot{n}= & \phi_{n}\left[n_{\infty}-n\right] / \tau_{n}=0
\end{aligned}
$$

where $m_{\infty}, a_{\infty}, n_{\infty}$ and $\tau_{\infty}$ are all functions of $V$, as they are defined in the Appendix. $g_{L}, g_{N a}, g_{K}, g_{A}, E_{L}, E_{N a}, E_{K}$ and $\phi_{n}$ are all constants. $b_{c}$ denotes the variable $b$ fixed as a constant. By definition, intersections of the nullclines provide the equilibria, i.e., Steady States of the system. The stability of an equilibrium point $\left(V_{s s}, n_{s s}\right)$ can be evaluated by analysing the Jacobian matrix of the system $\dot{V}$ and $\dot{n}$ at that point (Izhikevich 2007).

As shown in Fig. 5, in all of the conditions, the variable $b$ is no larger than 0.06 . Therefore, it is sufficient to evaluate $b_{c}<0.06$. The obtained phase spaces under different conditions are illustrated in Fig. 6. It can be seen from Fig. 6(a) that when $b_{c}=0$, the $V$ - and $n$-nullclines (denoted by solid and dished lines, respectively) have only one intersection, the system is unstable and a stable limit cycle attractor (corresponding to repetitive spiking) exists. In such case, the system will converge onto the limit cycle from any initial state. As a result, the membrane potential will keep changing along the limit cycle without termination (Fig. 6(a), bold line). However, it has been shown in Fig. 5 that with low membrane potentials, the gating variable $b$ can quickly increase from zero. Such increase shifts the lower deflection point of the $V$-nullcline downwards but does not change the $n$-nullcline. As a result, the $V$-nullcline additionally intersects with the $n$-nullcline at the low-voltage range and forms a new equilibrium at $b_{c}=0.027$ (Fig. 6(b)). At this critical point, the equilibrium is actually a coalescence of a stable node (corresponding to a stable steady-state) and an unstable equilibrium (saddle). With further increase in $b$, the node and saddle split, and the system now has a stable steady state (Fig. 6(c)). In this case, the neuron cannot produce repetitive spiking as trajectories of neuronal dynamics always terminate at the stable steady-state, as shown in Fig. 6(c). This procedure is referred to as a saddle-node bifurcation, which results in a transition of the system from periodic spiking to resting dynamics. This explains the trajectories illustrated in Fig. 5(a and b). With low initial membrane potential, the gating variable $b$ quickly activates and the bifurcation occurs, which produces a stable node. The dynamical trajectory is attracted by the stable node without spiking (Fig. 5(a)). However, with a high initial membrane potential, the gate $b$ is kept inactive at the beginning. At this moment, the system only has a stable limit cycle. The system's trajectory is attracted by this limit cycle and produces a spike. At the end of the spike, the gate $b$ activates which leads to a bifurcation. The trajectory is then attracted to the stable node (Fig. 5(b)). When $g_{A}$ is sufficiently low, the $V$-nullcline is prevented 


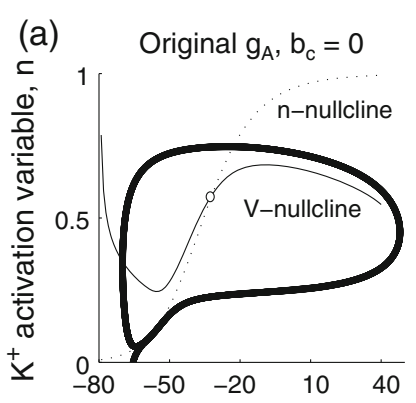

(b) Original $g_{A}, b_{c}=0.027$,

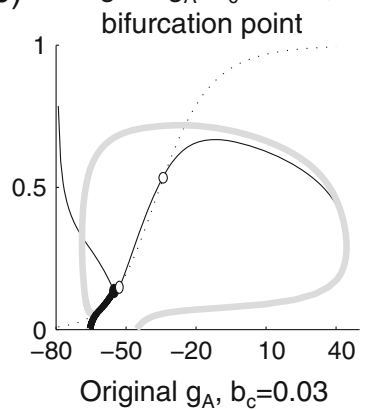

(c)

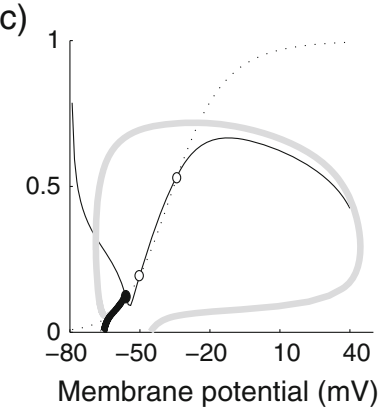

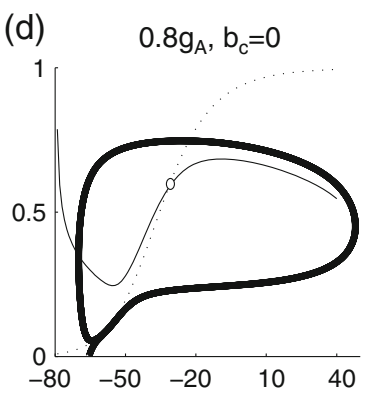

(e) $\quad 0.8 g_{A}, b_{c}=0.027$

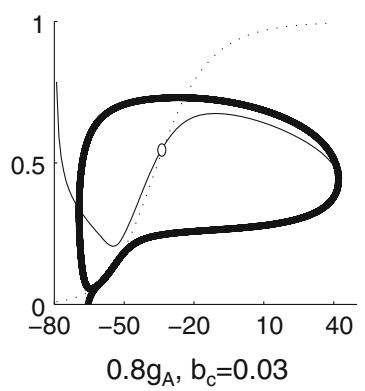

(f)

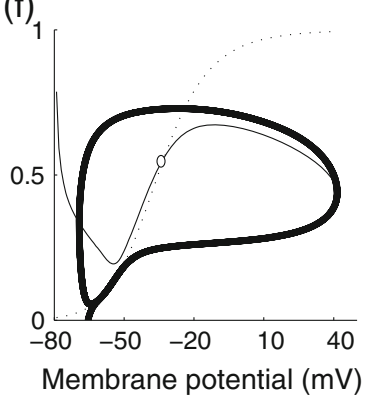

Fig. 6 Phase spaces of $V-n$ with $b$ fixed at different constants in various conditions. Plots (a), (b) and (c) are obtained in the original $\mathrm{g}_{\mathrm{A}}$ condition and Plots (d), (e) and (f) are obtained in the $0.8 \mathrm{~g}_{\mathrm{A}}$ condition. Filled circles denote stable equilibria, and opened circles denote unstable equilibria. The trajectories are denoted by bold black lines (initial membrane potentials being $-65 \mathrm{mV}$ ) and bold grey lines (initial membrane potentials being $-45 \mathrm{mV}$ ). In the original $\mathrm{g}_{\mathrm{A}}$ condition, bifurcation, induced by increasing $b$, produces a stable steady state. Therefore, the excitability of the system (tendency to spike) is inhibited. In the $0.8 \mathrm{~g}_{\mathrm{A}}$ condition, no bifurcation happens such that the excitability is enhanced

from shifting downwards due to the increase in $b$. As a result, the saddle-node bifurcation may not happen with the increase in $b$ (Fig. 6(d, e and $\mathrm{f}$ )). In such cases, the system only has a limit cycle for various values of $b$, which explains the trajectories demonstrated in Fig. 5(c and d).

\subsubsection{Hyper neuronal excitability can cause the disappearance of theta rhythm}

In this section, the relationship between the increased neuronal excitability and the decreased theta band power is investigat-

ed. The system's oscillation is investigated using the full network in a noise-free condition. As shown in Fig. 3, the pyramidal neurons activate in phase relative to a theta cycle. We also find that with very low $g_{A}$, the pyramidal and OLM neurons spike in phase, while MSGABA and basket neurons do not spike at all. Therefore, measuring the oscillation frequency of pyramidal neurons is sufficient to provide an approximation of the network oscillation. Figure 7(a) shows the oscillation frequency of the pyramidal neurons in the noise-free condition. It can be seen that there exists a critical point corresponding to the value of $g_{A}$ between $0.3-0.4$ times its normal value. At larger values of $g_{A}$, the pyramidal neurons oscillate at the theta rhythm (Fig. 7(a) left and middle insets). But at smaller values of $g_{A}$, theta oscillations abruptly disappear with the pyramidal neurons spiking at a high frequency (Fig. 7(a) right inset), which gradually increases with decreasing $\mathrm{g}_{\mathrm{A}}$. The slight frequency drop with 0.5 and $0.4 \mathrm{~g}_{\mathrm{A}}$ is due to the pyramidal neurons producing multiple spikes around the peak of the theta cycle caused by high excitability (Fig. 7(a) middle inset), which expand the oscillation period and decrease the frequency. At this time, theta frequency and high frequency co-exist in the network oscillation. Figure 7(b) shows an example of the transition of the dominant frequency with $g_{A}$ decrease.

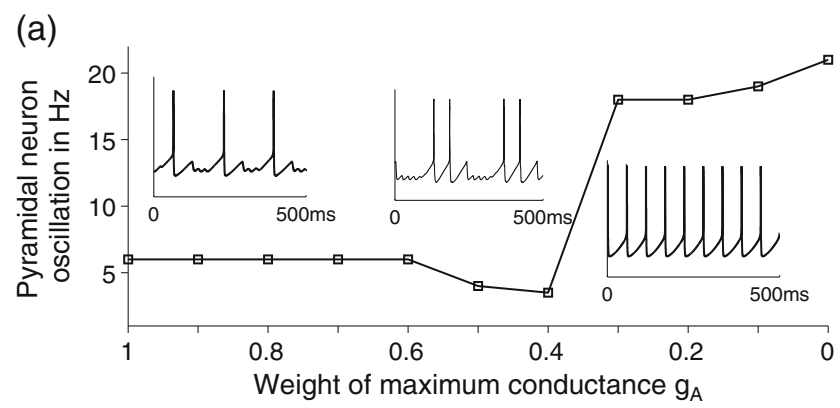

(b)

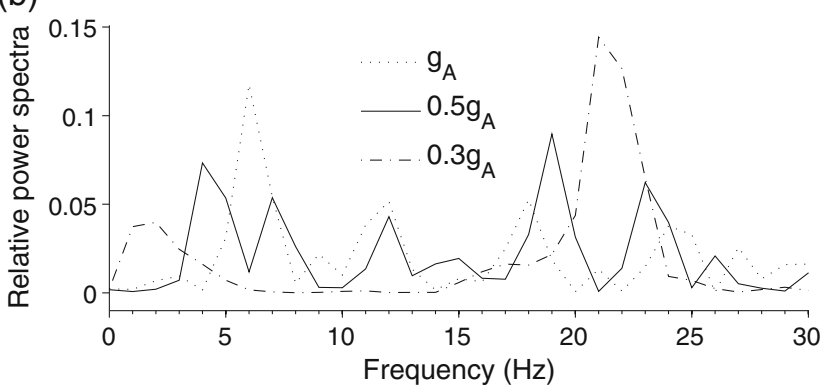

Fig. 7 Oscillation frequency increases with decreasing $g_{A}$ (theta band power disappearance). (a) Oscillation frequencies of a pyramidal neuron with different weights of $g_{A}$ in the noise-free condition. Note the abrupt transition in frequency at $0.3-0.4 \mathrm{~g}_{\mathrm{A}}$. Insets: dynamics of a pyramidal neuron oscillating in theta (left and middle) and higher (right) frequencies. (b) Power spectra peaks with different $g_{A}$ values in the noisy condition. The higher frequency band peak increases at the expense of the theta band peak as $g_{A}$ decreases 
It has been shown in Fig. 6 that with $g_{A}$ decreasing, the lower deflection point of the $V$-nullcline is prevented from shifting towards the $n$-nullcline. The lower $g_{\mathrm{A}}$ is, the greater the distance from the $V$-nullcline to the $n$-nullcline will be. As a result, the depolarization of the pyramidal neurons becomes faster (Guckenheimer and Holmes 1997; Izhikevich 2007). The decreased $g_{A}$ induced faster depolarization is also illustrated in Fig. 8(b). When $\mathrm{g}_{\mathrm{A}}$ is as low as 0.3 times its original value, the pyramidal neurons will always depolarize faster than the basket neurons, which results in relatively earlier spiking of pyramidal neurons compared to that of basket neurons, thus swopping their phase relationship within a theta cycle. This will evoke the spikes of the OLM neurons via AMPA and NMDA, which prevents the

(a)
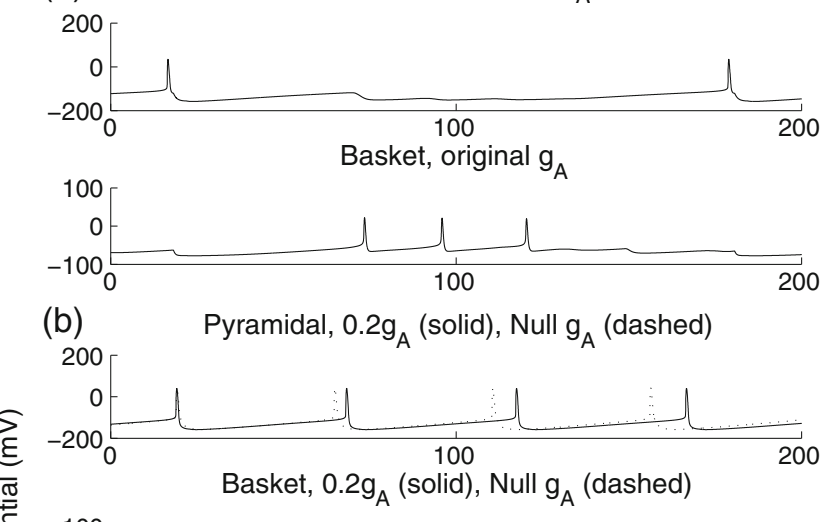

100

0

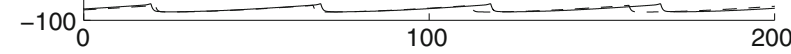

(c) Pyramidal, original $g_{A}$ with periodic current pulse
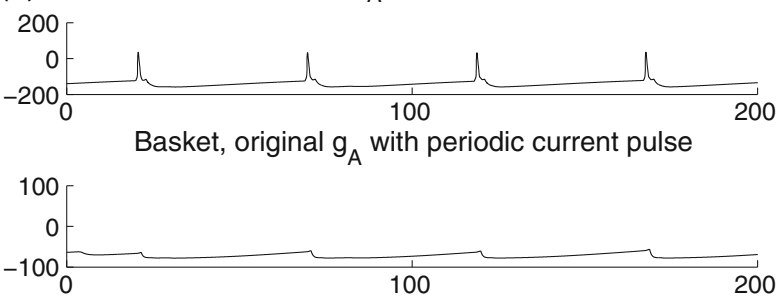

Periodic depolarization current pulse applied on pyramidal neurons

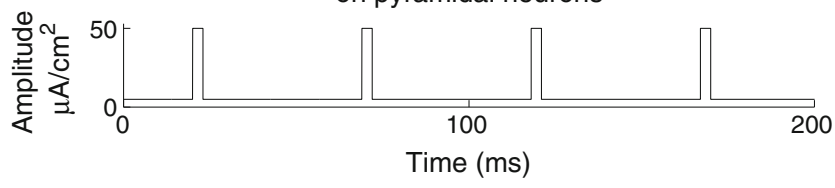

Fig. 8 Membrane potential dynamics of pyramidal and basket neurons. (a) the original $\mathrm{g}_{\mathrm{A}}$ condition; (b) $0.2 \mathrm{~g}_{\mathrm{A}}$ condition (solid line) and null $\mathrm{g}_{\mathrm{A}}$ condition (dashed line), the initial spike of the dashed line is aligned to the solid line; $(\mathbf{c})$ original $\mathrm{g}_{\mathrm{A}}$ condition with artificial brief current pulses. This figure indicates that the disappearance of theta oscillation with very low $g_{A}$ is due to the swopping of spiking phase of pyramidal and basket neurons caused by the fast depolarization of the pyramidal neurons basket neurons from spiking. Without inhibition from the basket neurons, the pyramidal neurons will depolarize quickly again and produce more spikes. As a result, the pyramidal neurons will consecutively spike at a higher frequency and the network oscillation will only be dependent on the spiking of the pyramidal neurons, but not on the spiking phase relationships among the different neuronal populations. As the depolarization speed of the pyramidal neurons keeps increasing with decreasing $\mathrm{g}_{\mathrm{A}}$, the oscillation frequency of pyramidal neurons increases slightly.

To verify our hypothesis on the causal relationship between changes in the pyramidal neurons and basket neurons, we artificially injected brief depolarization current pulses into the pyramidal neurons just before the spikes of the basket neurons in the noise-free original $g_{A}$ condition. Given our hypothesis, we would expect similar results to those obtained under the very low $\mathrm{g}_{\mathrm{A}}$ condition. A brief current pulse with a duration of $3 \mathrm{~ms}$ and amplitude of $50 \mu \mathrm{A} / \mathrm{cm}^{2}$ was applied every $55 \mathrm{~ms} .200 \mathrm{~ms}$ simulation results selected from single-trials are presented in Fig. 8(c). As anticipated, membrane potential dynamics observed in the original $g_{A}$ condition were very similar to those observed in the very low $\mathrm{g}_{\mathrm{A}}$ condition.

\section{Discussion}

In our previous work (Zou et al. 2011), we have investigated the impact of $A \beta$-induced changes in various ionic channels of hippocampal pyramidal neurons on system dynamics by evaluating theta band power changes. We have found that only $A \beta$ blocked $I_{A}$ can significantly alter theta band power. In this paper, we explored a wider range of the model parameters of $I_{A}$ and found $A \beta$ blocked $\mathrm{I}_{\mathrm{A}}$ induced both increase and decrease in theta band power. Both the theta band power changes were induced by the enhanced neuronal excitability. Analysis based on a reduced pyramidal neuronal model showed that the decreased $\mathrm{I}_{\mathrm{A}}$ prevented the emergence of a steady state of the neuron and in turn increased the neuronal excitability. As we have shown in Fig. 3, the network theta oscillation consisted of various neuronal populations spiking at different phases of a theta cycle. Supposing a theta cycle starts from the spiking of pyramidal neurons, the spiking phase of different neuronal populations within the theta cycle should be in the temporal order: the pyramidal and OLM neurons, followed by the basket neurons, and finally the MSGABA neurons. With moderate low $\mathrm{g}_{\mathrm{A}}$, such spiking relationship was kept; only more pyramidal neurons produced spikes during the peak of pyramidal theta oscillation, which increased the synchrony of the 
pyramidal population and thus increased the theta band power. However, when the pyramidal neurons became hyper-excited, the spiking phase relationship was violated and the theta band power decreased. Interestingly, the decreased theta band power is consistent with an experimental finding on animal model of AD (Mugantseva and Podolski 2009).

As the hippocampo-septal theta rhythm plays an important role in memory processing, our finding may provide explanations for the memory deficits observed in $\mathrm{AD}$ patients and animal models. A simulation demonstration of the low $\mathrm{g}_{\mathrm{A}}$ induced memory deficits is illustrated in

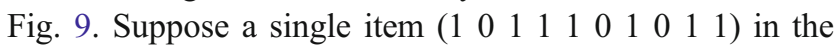
memory is represented by neuronal dynamics over all the 10 pyramidal neurons, by depolarizing the selective neurons. Then, under the control condition, the memory can be correctly presented over time by the periodic spiking patterns of the pyramidal neurons (Fig. 9(a)). With lower $\mathrm{g}_{\mathrm{A}}$, undesired neurons are also incorporated into spiking, possibly resulting in either a wrong memory or multiple interfering memories being represented (Fig. 9(b)). With very low $g_{A}$, all pyramidal neurons become hyper-excited and the theta rhythm ceases to exist (Fig. 9(c)). Indeed, a recent study has shown that $A \beta$ could be the main cause of epilepsy in $\mathrm{AD}$ due to hippocampal network hyperexcitability (Palop et al. 2007). Our work supports such an observation.

(a)

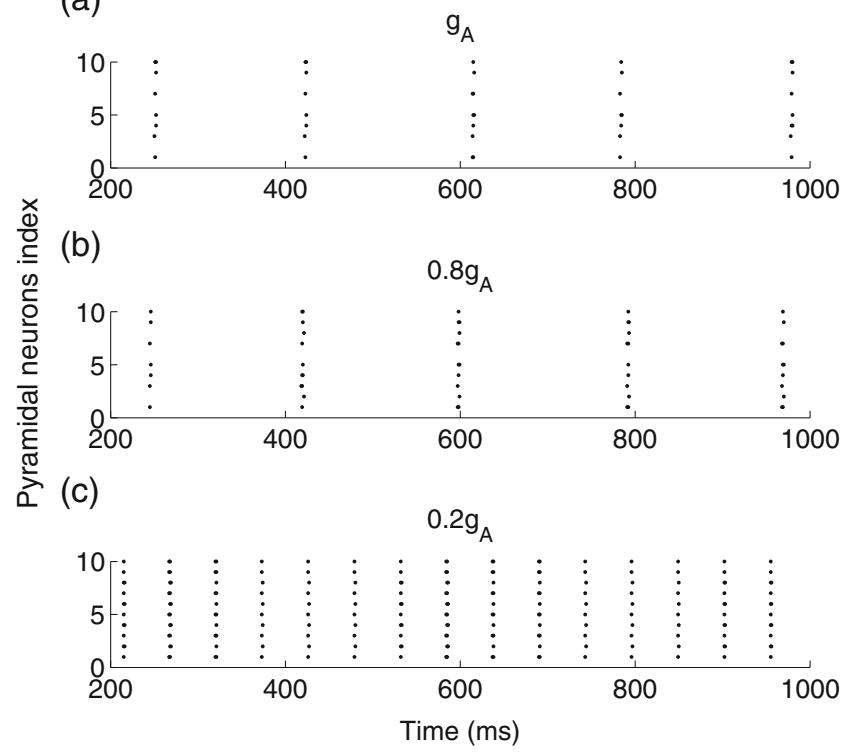

Fig. 9 A hypothetical demonstration of low $\mathrm{I}_{\mathrm{A}}$ on memory deficits and epilepsy. (a) The correct memory pattern is represented in the original $\mathrm{g}_{\mathrm{A}}$ condition. (b) and (c) show the memory representation is affected by the low $g_{A}$ induced high neural excitability. The hyper-excited pyramidal neurons in (c) may also be the cause of epilepsy in $\mathrm{AD}$
With regard to the mechanisms underlying theta rhythm generation in the hippocampus, there exist multiple hypotheses. One theory hypothesized that the Medial Septem GABAergic afference imposes the theta oscillation on the GABAergic cells in the hippocampus and in turn on the firing of pyramidal neurons. This hypothesis has been supported by experimental and theoretical studies (Stewart and Fox 1990; Cobb et al. 1995; Wang 2002; Freund and Antal 1988; Toth et al. 1997). However, some other studies showed that the slow deactivation current $I_{h}$ in the pyramidal neurons and OLM neurons may also contribute to theta oscillation by providing rebound action potentials (Rotstein et al. 2005; Orban et al. 2006). Orban et al. (2006) have demonstrated that $I_{h}$ in the pyramidal neurons act as a pacemaker of the theta rhythm in a hippocampus standalone network. In our model, we followed the former hypothesis, i.e., hippocampo-septal theta oscillation is originated from MSGABA neurons. Furthermore, there is no experimental evidence showing that $I_{h}$ in the hippocampus is affected by $A \beta$. Therefore we have excluded the $I_{h}$ in the pyramidal neurons. Nevertheless, the comparison of the effects of these two hypotheses will be an interesting project on its own, but it is beyond the scope of this paper. In our work, the simulations and analyses were based on an all-to-all network connectivity, as heterogeneity in the neuronal connections may cause some difficulties in our analysis. For example, it will be more difficult to sort, evaluate and compare the depolarization time of various neurons. Nevertheless, as shown in (Wang and Buzsaki 1996; Golomb and Hansel 2000), as long as the number of synapses per cell is larger than a critical value, the neuronal network dynamics remain robust.

To conclude, we have shown that $\mathrm{A} \beta$ blockage of $\mathrm{I}_{\mathrm{A}}$ could be an important reason for causing behavioural changes in the hippocampal network. Although in this work, we only focus on the system dynamical changes caused by the pathological changes in the hippocampal pyramidal neurons, it should be admitted that the $A \beta$-indued hippocampal dysfunction may be more complex. For example, it has been shown in (Villette et al. 2010; Colom et al. 2010) that A $\beta$ induced GABAergic and cholinergic system degeneration in the medial septum may also cause hippocampal dysfunctions. GABAergic neurons inhibit hippocampal OLM and basket neurons and act as a hippocampal theta rhythm pacemaker and cholinergic neurons control the acetylcholine level in the hippocampus, which can change the dynamics of pyramidal neurons and synapses. Investigating these factors will be a topic of future research.

Acknowledgement This study is currently supported under the CNRT award by the Northern Ireland Department for Employment and Learning through its "Strengthening the All-Island Research Base" initiative. We are grateful to Dr. Christian Hölscher for comments on an earlier version of our manuscript. 


\section{Appendix}

The membrane capacitance $C=1 \mu F / \mathrm{cm}^{2}$ for all of the follow equations, therefore it will be ignored. The $\tau$ in $(m s) ; E$ and $V$ in $(\mathrm{mV}) ; I$ in $\left(\mu \mathrm{A} / \mathrm{cm}^{2}\right) ; \mathrm{g}$ in $\left(\mathrm{mS} / \mathrm{cm}^{2}\right) ; \alpha$ and $\beta$ in $\left(\mathrm{ms}^{-1}\right) ; K$ in $(\mu M)$; B in $\left(\mu M(m s \mu A)^{-1} \mathrm{~cm}^{2}\right)$ and the rest are dimensionless constant. We used an Euler method for numerically integrating the stochastic differential equations, using a time step of $0.01 \mathrm{~ms}$. Smaller time steps do not change our results.

\section{Neuronal dynamics}

The pyramidal somatic and dendritic membrane potentials, denoted by $V_{s}$ and $V_{d}$, obtains the following equations:

$\dot{V}_{s}=-I_{L}-I_{N a}-I_{K}-I_{C a}-I_{A}-I_{C T}-\frac{g_{c}}{p}\left(V_{s}-V_{d}\right)-I_{s y n, s}+I$

$$
\begin{aligned}
\dot{V}_{d}= & -I_{L}-I_{C a}-I_{A H P}-I_{A}-I_{C T}-\frac{g_{c}}{1-p} \\
& \times\left(V_{d}-V_{s}\right)-I_{\text {syn }, d}
\end{aligned}
$$

Where $g_{c}=2 \mathrm{mS} / \mathrm{cm}^{2}$ is the coupling conductance between soma and dendrite, $p=$ somatic area/total area $=$ 0.5. $I$ is the injected DC current and $I_{s y n}$ is the synaptic currents. $I_{L}=g_{L}\left(V-E_{L}\right)$. In our work, all of the ionic currents are modelled by the Hodgkin-Huxley type formalism, thus the dynamic of a gating variable $x$ satisfies first-order kinetics,

$\dot{x}=\phi_{x}\left[\alpha_{x}(1-x)-\beta_{x} x\right]=\phi_{x}\left[x_{\infty}-x\right] / \tau_{x}$

This equation will be used to calculate all of the gating

\begin{tabular}{|c|c|c|}
\hline Channel & Definition & Parameters \\
\hline$I_{N a}$ & $g_{N a} m_{\infty}^{3} h\left(V-E_{N a}\right)$ & $\begin{array}{l}m_{\infty}=\alpha_{m} /\left(\alpha_{m}+\beta_{m}\right) \\
\alpha_{m}=-0.1(V+33) / \exp [-0.1(V+33)-1] \\
\beta_{m}=4 \exp [-(V+58) / 12] \\
\alpha_{h}=0.07 \exp [-(V+50) / 10] \\
\beta_{h}=1 / \exp [-0.1(V+20)+1]\end{array}$ \\
\hline$I_{K}$ & $g_{K} n^{4}\left(V-E_{K}\right)$ & $\begin{array}{l}\alpha_{n}=-0.01(V+34) / \exp [-0.1(V+34)-1] \\
\beta_{n}=0.125 \exp [-(V+44) / 25]\end{array}$ \\
\hline$I_{C a}$ & $g_{C a} m_{\infty}\left(V-E_{C a}\right)$ & $m_{\infty}=1 / \exp [-(V+20) / 9]$ \\
\hline$I_{A H P}$ & $g_{A H P}\left[\mathrm{Ca}^{2+}\right] /\left(\left[\mathrm{Ca}^{2+}\right]+K_{D}\right)\left(V-E_{K}\right)$ & $\begin{array}{l}\frac{a\left[\mathrm{Ca}^{2}\right]}{d t}=-\left[\mathrm{Ca}^{2+}\right] / \tau_{C a}-B I_{C a} \\
\tau_{C a}=1000, B=0.002, K_{D}=30 \mu M\end{array}$ \\
\hline$I_{A}$ & $g_{A} a^{3} b\left(V-E_{K}\right)$ & $\begin{aligned} \alpha_{a} & =-0.05(V+20) /\{\exp [-(V+20) / 15]-1\} \\
\beta_{a} & =0.1(V+10) /\{\exp [(V+10) / 8]-1\} \\
\alpha_{b} & =0.00015 / \exp [(V+18) / 15] \\
\beta_{b} & =0.06 /\{\exp [-(V+73) / 12]+1\}\end{aligned}$ \\
\hline$I_{C T}$ & $g_{C T} c^{2} d\left(V-E_{K}\right)$ & $\begin{array}{l}\alpha_{c}=-0.0077\left(V+V_{\text {shift }}+103\right) / \\
\left\{\exp \left[-\left(V+V_{\text {shift }}+103\right) / 12\right]-1\right\} \\
\beta_{c}=0.91-\alpha_{c} \\
\alpha_{d}=1 / \exp [(V+79) / 10] \\
\beta_{d}=4 /\{\exp [-(V-82) / 27]+1\} \\
V_{\text {shift }}=40 \log \left(\left[C a^{2+}\right] / 13.805\right) \\
\tau_{C a}=0.9, B=0.06\end{array}$ \\
\hline
\end{tabular}
variables.
The values of the other parameters are $\phi=4, g_{L}=0.1$ and $g_{C a}=0.5$ for soma and dendrite, $g_{N a}=45, g_{K}=18, g_{A}=20$ $g_{C T}=140$ and $g_{h}=0.01$ for soma and $g_{A H P}=5, g_{A}=60 g_{C T}=$ 70 and $g_{h}=0.02$ for dendrite; $E_{L}=-65, E_{N a}=55, E_{K}=-80$, $E_{C a}=120$, and $I_{\mu}=4.9$.
The OLM neuron is described as a single compartment model,

$\dot{V}=-I_{L}-I_{N a}-I_{K}-I_{C a}-I_{h}-I_{A H P}-I_{s y n}+I$ 


\begin{tabular}{lll}
\hline Channel & Definition & Parameters \\
$I_{N a}$ & $g_{N a} m_{\infty}^{3} h\left(V-E_{N a}\right)$ & $\alpha_{m}=-0.1(V+35) / \exp [-0.1(V+35)-1]$ \\
& & $\beta_{m}=4 \exp [-(V+60) / 18]$ \\
& $\alpha_{h}=0.07 \exp [-(V+58) / 20]$ \\
& $\beta_{h}=1 / \exp [-0.1(V+28)+1]$ \\
& & $\alpha_{n}=-0.01(V+34) / \exp [-0.1(V+34)-1]$ \\
$\mathrm{I}_{\mathrm{K}}$ & $g_{K} n^{4}\left(V-E_{K}\right)$ & $\beta_{n}=0.125 \exp [-(V+44) / 80]$ \\
& & $m_{\infty}=1 /\{\exp [-(V+20) / 9]+1\}$ \\
$\mathrm{I}_{\mathrm{Ca}}$ & $g_{C a} m_{\infty}^{2}\left(V-E_{C a}\right)$ & $\frac{d\left[C a^{++}\right]}{d t}=-\left[C a^{2+}\right] / \tau_{C a}-B I_{C a}$ \\
$\mathrm{I}_{\mathrm{AHP}}$ & $g_{A H P}\left[C a^{2+}\right] /\left(\left[C a^{2+}\right]+K_{D}\right)\left(V-E_{K}\right)$ & $\tau_{C a}=80, B=0.002, K_{D}=30 \mu M$ \\
& & $H_{\infty}=1 /\{\exp [(V+80) / 10]+1\}$ \\
$\mathrm{I}_{\mathrm{h}}$ & $g_{h} H\left(V-E_{h}\right)$ & $\left.\tau_{H}=200 /\{\exp [(V+70) / 20]\}+\exp [-(V+70) / 20]+5\right\}$ \\
& &
\end{tabular}

The other parameters are $\phi=5, g_{L}=0.1, g_{N a}=35, g_{K}=9$, $g_{A H P}=10, g_{C a}=1, g h=0.15 ; E_{L}=-65, E_{N a}=55, E_{K}=-90$, $E_{C a}=120, E_{h}=-40, I_{\mu}=0$.

The basket neuron is described as a single compartment model,

$\dot{V}=-I_{L}-I_{N a}-I_{K}-I_{s y n}+I$
The parameters for calculation of the ionic currents are the same as that of OLM and $I_{\mu}=1.4$.

The MSGABA neuron is described as a single compartment model,

$\dot{V}=-I_{L}-I_{N a}-I_{K}-I_{K S}-I_{s y n}+I$

\begin{tabular}{lll}
\hline Channel & Definition & Parameters \\
$\mathrm{I}_{\mathrm{Na}}$ & $g_{\mathrm{Na}} m_{\infty}^{3} h\left(V-E_{N a}\right)$ & $\beta_{m}=4 \exp [-(V+58) / 18]$ \\
& $\alpha_{h}=0.07 \exp [-(V+51) / 10]$ \\
& $\beta_{h}=1 / \exp [-0.1(V+21)+1]$ \\
& $\alpha_{n}=-0.01(V+38) / \exp [-0.1(V+38)-1]$ \\
$\mathrm{I}_{\mathrm{K}}$ & $\beta_{n}=0.125 \exp [-(V+48) / 80]$ \\
& $p_{\infty} n^{4}\left(V-E_{K}\right)$ & $\tau_{p}=6$ \\
$\mathrm{I}_{\mathrm{KS}}$ & $q_{\infty}=1 /\{\exp [-(V+34) / 6.5]+1\}$ \\
& $\tau_{q}=\tau_{q 0}(1+1 / \exp [(V+65) / 6.6]+1\}$ \\
& $\tau_{q 0}=100$ \\
& \\
&
\end{tabular}

The other parameters are $\phi=5, g_{L}=0.1, g_{N a}=50, g_{K}=8$, $g_{K S}=12 ; E_{L}=-50, E_{N a}=55, E_{K}=-85, I_{\mu}=22$.

Synaptic connection definition

There are three types of synaptic neurotransmitters, the inhibitory $\mathrm{GABA}_{\mathrm{A}}$, the excitatory NMDA and
AMPA. The $\mathrm{GABA}_{\mathrm{A}}$ inhibitory post synaptic current (IPSC) is described as $I_{G A B A_{A}}=g_{s y n} s\left(V-E_{G A B A_{A}}\right)$, where the activation variable $s$ is calculated by $s=\alpha F\left(V_{\text {pre }}\right)(1-s)-\beta s$. The $V_{\text {pre }}$ is the presynaptic neuron membrane potential, $F\left(V_{\text {pre }}\right)=1 /[1+\exp$ $\left.\left(-V_{\text {pre }} / K\right)\right]$. Parameters for different neurons couples are: 


\begin{tabular}{lc}
\hline basket-pyramidal & $\alpha=10, \beta=0.1, K=2, E_{G A B A_{A}}=-80$, \\
(b-p) & $g_{\text {sym }}=2.76$ \\
OLM-basket & $\alpha=20, \beta=0.1, K=2, E_{G A B A_{A}}=-80$, \\
(o-b) & $g_{\text {sym }}=1.76$ \\
OLM-pyramidal & $\alpha=20, \beta=0.1, K=2, E_{G A B A_{A}}=-85$, \\
(o-p) & $g_{\text {sym }}=1.76$ \\
OLM-MSGABA & $\alpha=20, \beta=0.1, K=0.5, E_{G A B A_{A}}=-80$, \\
(o-m) & $g_{\text {sym }}=0.5$ \\
basket-basket & $\alpha=10, \beta=0.1, K=2, E_{G A B A_{A}}=-75$, \\
(b-b) & $g_{\text {sym }}=0.125$ \\
MSGABA-OLM & $\alpha=10, \beta=0.1, K=2, E_{G A B A_{A}}=-75$, \\
(m-o) & $g_{\text {sym }}=0.5$ \\
MSGABA-MSGABA & $\alpha=10, \beta=0.1, K=2, E_{G A B A_{A}}=-75$, \\
(m-m) & $g_{\text {sym }}=0.25$ \\
MSGABA-basket & $\alpha=10, \beta=0.1, K=2, E_{G A B A_{A}}=-75$, \\
(m-b) & $g_{\text {sym }}=1$ \\
\hline
\end{tabular}

The AMPA and NMDA excitatory post synaptic current (EPSP) are described as $I_{A M P A}=g_{A M P A} S\left(V-E_{A M P A}\right)$ and $I_{N M D A}=g_{N M D A} B(V) s\left(V-E_{N M D A}\right)$, respectively. $s$ is updated as $s=\alpha[T](1-s)-\beta s, \quad[T]=T_{\max } /[1+\exp$ $\left.\left(-V_{\text {pre }}+V_{p}\right) / K_{p}\right], V_{p}=2, V_{K}=5, B(V)$ is calculated by $B(V)=1 /\left\{1+\exp (-0.062 V)\left[M g^{2+}\right] / 3.5\right\}$, where $\left[M g^{2}\right.$ $\left.{ }^{+}\right]=1 \mathrm{mM}$ by default. The $\alpha$ and $\beta$ for AMPA. and NMDA are $\alpha=1.1, \beta=0.19$ and $\alpha=0.072, \beta=0.0066$, respectively. $E_{A M P A}=E_{N M D A}=0$. From pyramidal to basket neurons $g_{A M P A}=0.1$, and from pyramidal to OLM neurons $g_{A M P A}=$ 1.35 and $g_{A M P A}=0.625$. The summated synaptic current is normalized by the number of presynaptic neurons.

\section{References}

Adeli, H., Ghosh-Dastidar, S., \& Dadmehr, N. (2005). Alzheimer's disease and models of computation: imaging, classification, and neural models. Journal of Alzheimer's Disease, 7(3), 187-199. discussion 255-162.

Chen, C. (2005). beta-Amyloid increases dendritic Ca2+ influx by inhibiting the A-type $\mathrm{K}+$ current in hippocampal CA1 pyramidal neurons. Biochemical and Biophysical Research Communications, 338(4), 1913-1919.

Chi, S., \& Qi, Z. (2006). Regulatory effect of sulphatides on BKCa channels. British Journal of Pharmacology, 149(8), 1031-1038.

Cobb, S. R., Buhl, E. H., Halasy, K., Paulsen, O., \& Somogyi, P. (1995). Synchronization of neuronal activity in hippocampus by individual GABAergic interneurons. Nature, 378(6552), 75-78. doi: $10.1038 / 378075 \mathrm{a} 0$.

Colom, L. V. (2006). Septal networks: relevance to theta rhythm, epilepsy and Alzheimer's disease. Journal of Neurochemistry, 96 (3), 609-623. doi:10.1111/j.1471-4159.2005.03630.x.

Colom, L. V., Castaneda, M. T., Banuelos, C., Puras, G., GarciaHernandez, A., Hernandez, S., et al. (2010). Medial septal betaamyloid 1-40 injections alter septo-hippocampal anatomy and function. Neurobiology of Aging, 31(1), 46-57. doi:10.1016/j. neurobiolaging.2008.05.006.
Csicsvari, J., Hirase, H., Czurko, A., Mamiya, A., \& Buzsaki, G. (1999). Oscillatory coupling of hippocampal pyramidal cells and interneurons in the behaving Rat. Journal of Neuroscience, 19(1), 274-287.

Cutsuridis, V., Cobb, S., \& Graham, B. P. (2010). Encoding and retrieval in a model of the hippocampal CA1 microcircuit. Hippocampus, 20(3), 423-446. doi:10.1002/hipo.20661.

Freund, T. F., \& Antal, M. (1988). GABA-containing neurons in the septum control inhibitory interneurons in the hippocampus. Nature, 336(6195), 170-173. doi:10.1038/336170a0.

Freund, T. F., \& Buzsaki, G. (1996). Interneurons of the hippocampus. Hippocampus, 6(4), 347-470. doi:10.1002/(SICI)1098-1063 (1996)6:4<347::AID-HIPO1>3.0.CO;2-I.

Golomb, D., \& Hansel, D. (2000). The number of synaptic inputs and the synchrony of large, sparse neuronal networks. Neural Computation, 12(5), 1095-1139.

Good, T. A., Smith, D. O., \& Murphy, R. M. (1996). Beta-amyloid peptide blocks the fast-inactivating $\mathrm{K}+$ current in rat hippocampal neurons. Biophysical Journal, 70(1), 296-304.

Guckenheimer, J., \& Holmes, P. (1997). Nonlinear oscillations, dynamical systems, and bifurcations of vector fields (Corr. 5th print. ed., Applied mathematical sciences, Vol. 42). New York: Springer.

Hajós, M., Hoffmann, W. E., Orbán, G., Kiss, T., \& Érdi, P. (2004). Modulation of septo-hippocampal Theta activity by GABAA receptors: an experimental and computational approach. Neuroscience, 126(3), 599-610.

Hardy, J. A., \& Higgins, G. A. (1992). Alzheimer's disease: the amyloid cascade hypothesis. Science, 256(5054), 184-185.

Hasselmo, M. E., Wyble, B. P., \& Wallenstein, G. V. (1996). Encoding and retrieval of episodic memories: role of cholinergic and GABAergic modulation in the hippocampus. Hippocampus, 6(6), 693-708. doi:10.1002/(SICI)1098-1063(1996)6:6<693::AID$\mathrm{HIPO} 12>3.0 . \mathrm{CO} ; 2-\mathrm{W}$.

Holscher, C., Gengler, S., Gault, V. A., Harriott, P., \& Mallot, H. A. (2007). Soluble beta-amyloid[25-35] reversibly impairs hippocampal synaptic plasticity and spatial learning. European Journal of Pharmacology, 561(1-3), 85-90. doi:10.1016/j. ejphar.2007.01.040.

Izhikevich, E. M. (2007). Dynamical systems in neuroscience: The geometry of excitability and bursting (Computational neuroscience). Cambridge: MIT Press.

Klausberger, T., \& Somogyi, P. (2008). Neuronal diversity and temporal dynamics: the unity of hippocampal circuit operations. Science, 321(5885), 53-57. doi:10.1126/science.1149381.

Klausberger, T., Magill, P. J., Marton, L. F., Roberts, J. D., Cobden, P. M., Buzsaki, G., et al. (2003). Brain-state- and cell-type-specific firing of hippocampal interneurons in vivo. Nature, 421(6925), 844-848. doi:10.1038/nature01374.

Li, X., Coyle, D., Maguire, L., Watson, D. R., \& McGinnity, T. M. (2010). Gray matter concentration and effective connectivity changes in Alzheimer's disease: a longitudinal structural MRI study. Neuroradiology. doi:10.1007/s00234-010-0795-1.

Minati, L., Edginton, T., Bruzzone, M. G., \& Giaccone, G. (2009). Current concepts in Alzheimer's disease: a multidisciplinary review. American Journal of Alzheimer's Disease and Other Dementias, 24(2), 95-121. doi:10.1177/1533317508328602.

Morse, T. M., Carnevale, N. T., Mutalik, P. G., Migliore, M., \& Shepherd, G. M. (2010). Abnormal excitability of oblique dendrites implicated in early alzheimer's: a computational study. Frontiers in Neural Circuits, 4. doi:10.3389/fncir.2010.00016.

Mugantseva, E. A., \& Podolski, L. Y. (2009). Animal model of Alzheimer's disease: characteristics of EEG and memory. Central European Journal of Biology, 4, 507-514.

Orban, G., Kiss, T., \& Erdi, P. (2006). Intrinsic and synaptic mechanisms determining the timing of neuron population activity 
during hippocampal theta oscillation. Journal of Neurophysiology, 96(6), 2889-2904. doi:10.1152/jn.01233.2005.

Palop, J. J., \& Mucke, L. (2010). Amyloid-beta-induced neuronal dysfunction in Alzheimer's disease: from synapses toward neural networks. Nature Neuroscience, 13(7), 812-818. doi:10.1038/ nn. 2583 .

Palop, J. J., Chin, J., Roberson, E. D., Wang, J., Thwin, M. T., BienLy, N., et al. (2007). Aberrant excitatory neuronal activity and compensatory remodeling of inhibitory hippocampal circuits in mouse models of Alzheimer's disease. Neuron, 55(5), 697-711. doi:10.1016/j.neuron.2007.07.025.

Pedroarena, C., \& Llinas, R. (1997). Dendritic calcium conductances generate high-frequency oscillation in thalamocortical neurons. Proceedings of the National Academy of Sciences of the United States of America, 94(2), 724-728.

Rotstein, H. G., Pervouchine, D. D., Acker, C. D., Gillies, M. J., White, J. A., Buhl, E. H., et al. (2005). Slow and fast inhibition and an H-current interact to create a theta rhythm in a model of CA1 interneuron network. Journal of Neurophysiology, 94(2), $1509-1518$.

Stewart, M., \& Fox, S. E. (1990). Do septal neurons pace the hippocampal theta rhythm? Trends in Neurosciences, 13(5), 163168.

Takahashi, R. H., Capetillo-Zarate, E., Lin, M. T., Milner, T. A., \& Gouras, G. K. (2010). Co-occurrence of Alzheimer's disease ssamyloid and tau pathologies at synapses. Neurobiology of Aging, 31(7), 1145-1152. doi:10.1016/j.neurobiolaging.2008.07.021.

Tiraboschi, P., Hansen, L. A., Thal, L. J., \& Corey-Bloom, J. (2004). The importance of neuritic plaques and tangles to the development and evolution of AD. Neurology, 62(11), 1984-1989.

Toth, K., Freund, T. F., \& Miles, R. (1997). Disinhibition of rat hippocampal pyramidal cells by GABAergic afferents from the septum. The Journal of Physiology, 500(Pt 2), 463-474.

Tran, M. H., Yamada, K., \& Nabeshima, T. (2002). Amyloid betapeptide induces cholinergic dysfunction and cognitive deficits: a minireview. Peptides, 23(7), 1271-1283.

Vertes, R. P. (2005). Hippocampal theta rhythm: a tag for short-term memory. Hippocampus, 15(7), 923-935. doi:10.1002/ hipo. 20118.

Villette, V., Poindessous-Jazat, F., Simon, A., Lena, C., Roullot, E., Bellessort, B., et al. (2010). Decreased rhythmic GABAergic septal activity and memory-associated theta oscillations after hippocampal amyloid-beta pathology in the rat. Journal of Neuroscience, 30(33), 10991-11003.

Wang, X.-J. (1998). Calcium coding and adaptive temporal computation in cortical pyramidal neurons. Journal of Neurophysiology, 79(3), 1549-1566.

Wang, X.-J. (2002). Pacemaker neurons for the theta rhythm and their synchronization in the septohippocampal reciprocal loop. Neurophysiology, 87, 889-900.

Wang, X.-J., \& Buzsaki, G. (1996). Gamma oscillation by synaptic inhibition in a hippocampal interneuronal network model. Journal of Neuroscience, 16(20), 6402-6413.

Warman, E. N., Durand, D. M., \& Yuen, G. L. (1994). Reconstruction of hippocampal CA1 pyramidal cell electrophysiology by computer simulation. Journal of Neurophysiology, 71(6), 20332045.

Webster, N. J., Ramsden, M., Boyle, J. P., Pearson, H. A., \& Peers, C. (2006). Amyloid peptides mediate hypoxic increase of L-type $\mathrm{Ca} 2+$ channels in central neurones. Neurobiology of Aging, 27 (3), 439-445.

Xu, C., Qian, C., Zhang, Z., Wu, C., Zhou, P., \& Liang, X. (1998). Effects of beta-amyloid peptide on transient outward potassium current of acutely dissociated hippocampal neurons in CA1 sector in rats. Chinese Medical Journal (English Edition), 111(6), 492-495.

Ye, H., Jalini, S., Mylvaganam, S., \& Carlen, P. (2010). Activation of large-conductance $\mathrm{Ca}(2+)$-activated $\mathrm{K}(+)$ channels depresses basal synaptic transmission in the hippocampal CA1 area in APP (swe/ind) TgCRND8 mice. Neurobiology of Aging, 31(4), $591-604$

Ylinen, A., Soltesz, I., Bragin, A., Penttonen, M., Sik, A., \& Buzsaki, G. (1995). Intracellular correlates of hippocampal theta rhythm in identified pyramidal cells, granule cells, and basket cells. Hippocampus, 5(1), 78-90.

Zhang, C. F., \& Yang, P. (2006). Zinc-induced aggregation of Abeta (10-21) potentiates its action on voltage-gated potassium channel. Biochemical and Biophysical Research Communications, 345(1), 43-49. doi:10.1016/j.bbrc.2006.04.044.

Zou, X., Coyle, D., Wong-Lin, K., \& Maguire, L. (2011). Computational study of hippocampal-septal theta rhythm changes due to beta-amyloid-altered ionic channels. PLoS One. 